\title{
ADESÃO A ORIENTAÇÕES FONOAUDIOLÓGICAS APÓS A ALTA DO TRATAMENTO VOCAL EM DOCENTES: ESTUDO PROSPECTIVO
}

\author{
Adherence to voice therapy guidelines after discharge from \\ vocal treatment in teachers: a prospective study
}

\author{
Ana Cristina Côrtes Gama (1), Viviane Souza Bicalho (2), Amanda Freitas Valentim (3), \\ lara Barreto Bassi ${ }^{(4)}$, Letícia Caldas Teixeira ${ }^{(5)}$, Ada Ávila Assunção ${ }^{(6)}$
}

\begin{abstract}
RESUMO
Objetivo: investigar a adesão a orientações fonoaudiológicas de professores da rede municipal de ensino que foram atendidos no Ambulatório de Voz do Hospital das Clínicas da Universidade Federal de Minas Gerais e que receberam alta da fonoterapia. Método: foi realizada entrevista por meio de telefone a 39 pacientes atendidos no período de agosto de 2007 a dezembro de 2008 no Ambulatório de Voz do Hospital das Clínicas da Universidade Federal de Minas Gerais. A entrevista visou analisar a adesão a orientações após alta fonoterápica. Resultados: os resultados desta pesquisa apontam que 34 pacientes $(87,1 \%)$ referem seguir as orientações fonoaudiológicas, quatro $(10,2 \%)$ seguem parcialmente e um $(2,5 \%)$ refere não seguir. Conclusão: os professores, em sua maioria, aderem às orientações fonoaudiológicas após alta fonoterápica. Os resultados sugerem que a terapia fonoaudiológica favorece a manutenção de um comportamento vocal saudável.
\end{abstract}

DESCRITORES: Voz; Distúrbios da Voz; Adesão do Paciente; Fonoaudiologia

\section{INTRODUÇÃO}

Professores são profissionais da voz e, por isso, estão expostos à grande demanda vocal. São os

(1) Fonoaudióloga; Professora associada do Departamento de Fonoaudiologia da Universidade Federal de Minas Gerais, UFMG, Belo Horizonte, Minas Gerais, Brasil; Doutora em Distúrbios da Comunicação Humana pela Universidade Federal de São Paulo.

(2) Fonoaudióloga graduada pela Universidade Federal de Minas Gerais, UFMG, Belo Horizonte, Minas Gerais, Brasil.

(3) Fonoaudióloga; Mestranda em Engenharia Mecânica pela Universidade Federal de Minas Gerais.

(4) Fonoaudióloga; Mestre em Saúde Coletiva pela Universidade Federal de Minas Gerais.

(5) Fonoaudióloga; Professora assistente do Departamento de Fonoaudiologia da Faculdade de Medicina da Universidade Federal de Minas Gerais, UFMG, Belo Horizonte, Minas Gerais, Brasil; Mestre em Educação pela Universidade Federal de Minas Gerais.

(6) Médica; Professora associada do Departamento de Medicina Preventiva e Social da Faculdade de Medicina da Universidade Federal de Minas Gerais, UFMG, Belo Horizonte, Minas Gerais, Brasil; Doutora em Ergonomia pela École Pratique Des Hautes Études - França.

Conflito de interesses: inexistente profissionais mais vulneráveis para a ocorrência de disfonia ${ }^{1}$ devido à falta de conhecimento sobre cuidados vocais e ao uso intenso da voz ${ }^{2}$.

Diante do diagnóstico de disfonia, os pacientes são encaminhados para tratamento fonoaudiológico. Espera-se do paciente adesão às orientações quanto à realização diária de exercícios de voz, produção vocal mais equilibrada além da eliminação de comportamentos vocais abusivos ${ }^{3}$.

$\mathrm{Na}$ prática clínica, a melhora dos sintomas e das alterações diagnosticadas indica a alta do paciente desde que o quadro esteja estabilizado. Nesse instante, o paciente será instruído quanto ao comportamento vocal saudável e quanto à importância dos exercícios de voz ${ }^{4}$.

A adesão do paciente ao tratamento, sobretudo, às orientações citadas anteriormente, não é automática. Segundo um estudo a "adesão ao tratamento é um processo multifatorial que se estrutura em uma parceria entre quem cuida e quem é cuidado; diz respeito, à freqüência, à constância e à perseverança na relação com o cuidado em busca da saúde" ${ }^{5}$. 
Vários estudos evidenciam a baixa adesão dos pacientes às orientações e à prescrição medicamentosa, independentemente do tipo de enfermidade crônica. Foi constatado que $53 \%$ dos pacientes hipertensos não aderem às mudanças orientadas no estilo de vida ${ }^{6}$. Apenas $45 \%$ dos pacientes que sofreram infarto agudo do miocárdio seguem corretamente a prescrição médica após um mês da alta hospitalar ${ }^{7}$. Em um estudo realizado com pacientes com Diabetes Mellitus (DM), foi verificado que 93,3\% estão satisfeitos com o atendimento, 92,9\% com o médico e $81,7 \%$ com a estrutura do serviço e acesso a consultas. A prevalência de não-adesão ao tratamento foi de $71 \%$ e a questão da satisfação do usuário mostrou-se como fator influente no grau de adesão ao tratamento ${ }^{8}$.

No tocante às disfonias, apesar da constatação na prática sobre a baixa adesão do paciente ao tratamento proposto, ainda há carência de aprofundamento sobre o problema.

Entre os raros estudos, uma pesquisa analisou a prevalência da adesão dos pacientes à indicação de terapia de voz e avaliou os fatores associados. As variáveis de interesse consideradas foram as seguintes: sexo, idade e raça/etnia; diagnóstico otorrinolaringológico e grau da disfonia. Foram examinados 147 prontuários de pacientes de duas instituições que procuraram um laringologista por queixas vocais e foram encaminhados para fonoterapia. Os resultados obtidos evidenciaram que apenas $35,4 \%$ pacientes iniciaram o tratamento e $64,6 \%$ não procuraram atendimento. Não houve relação estatisticamente significante entre as variáveis estudadas com a adesão à indicação do tratamento fonoterápico. Para explicar os resultados, os autores sugerem analisar fatores subjetivos como a motivação e disponibilidade para a mudança de comportamento ${ }^{9}$.

Um estudo com 15 pacientes demonstrou que os mesmos percebem como fatores importantes para a adesão à terapia de voz com o método de ressonância: motivação, comprometimento, aprendizado, auto-percepção vocal e um bom relacionamento com o terapeuta ${ }^{10}$.

Como a fonoterapia inclui a abordagem comportamental e depende intrinsecamente do envolvimento ativo do paciente, é possível que o fracasso na adesão ao tratamento identificado na prática clínica e nos raros estudos encontrados na literatura seja explicado pelas dificuldades relacionadas à mudança de comportamentos já cristalizados. Ademais, é lúcido supor que o paciente se depare com entraves educacionais e sociais para desenvolver os exercícios recomendados fora do ambiente terapêutico.
Estudos anteriores sobre a adesão ao tratamento levaram ao desenvolvimento de conceituações sobre a mudança dos comportamentos relacionados à saúde do indivíduo. Entre eles, o Modelo Transteórico (MTT) ${ }^{11}$ pode ser empregado no campo da fonoterapia e contribuir para o entendimento da mudança do comportamento e adesão à terapia instituída pelo fonoaudiólogo.

Segundo o MTT, a mudança de comportamento envolve cinco estágios, conceituados como pré-contemplação, estágio em que os indivíduos não estão dispostos a mudar de comportamento; estágio de contemplação, em que os indivíduos consideram a possibilidade de mudança de comportamento; estágio de preparação, no qual os pacientes se encontram mais preparados para realizar mudanças; estágio de ação, no qual os pacientes se comprometem a modificar comportamentos vocais e o último estágio, manutenção, no qual há integração do novo comportamento adotado e o paciente é capaz de manter a voz saudável ${ }^{11}$.

O objetivo desta pesquisa foi avaliar a adesão às orientações fonoaudiológicas em um grupo de professores da rede municipal de ensino que foram atendidos no Ambulatório de Voz do Hospital das Clínicas da Universidade Federal de Minas Gerais (AV-HC) e que receberam alta da fonoterapia.

\section{MÉTODO}

Trata-se de um estudo prospectivo de análise qualitativa. Foram estudados 39 professores acompanhados no AV-HC desde agosto/07 até dezembro/08. O estudo foi desenvolvido em duas etapas: a) levantamento de todos os casos registrados no período que estavam sendo atendidos no serviço a fim de identificar os professores que receberam alta após o tratamento realizado; b) análise de prontuário e entrevistas a 39 sujeitos incluídos na amostra após a análise dos dados obtidos à primeira etapa.

Todos os professores foram submetidos à terapia de voz combinada de forma direta e indireta $^{12}$. Os critérios de alta fonoaudiológica utilizados no AV-HC são os seguintes: ausência de queixa de fadiga vocal, qualidade vocal adaptada às condições anátomo-funcionais da laringe, avaliada por meio de avaliação perceptivo-auditiva e exames otorrinolaringológicos. Considera-se também, a presença de resistência vocal necessária para cumprir as exigências da demanda social e/ ou profissional do paciente.

Por meio da busca aos prontuários identificaramse 76 sujeitos, sendo que 40 receberam alta fonoaudiológica, 35 foram desligados por faltas conse- 
cutivas e/ou atrasos e houve um caso de suspensão do tratamento em decorrência de co-morbidade.

Não foi possível encontrar um paciente entre os 40 previamente selecionados. Os demais (39) concordaram em participar do estudo após o contato ao telefone realizado no período de dezembro de 2008 a fevereiro de 2009.

Foram coletados os seguintes dados que constavam nos prontuários: sexo, idade, escolaridade, tempo de docência, tipo de disfonia, tempo de tratamento e data da alta. O tipo de disfonia foi categorizado de acordo com a classificação de Behlau et al. (2001) ${ }^{13}$.

A amostra foi constituída de 39 sujeitos, sendo 36 do sexo feminino $(92,3 \%)$ e três do sexo masculino $(7,6 \%)$, com idade entre 23 e 57 anos (média 39,1; DP 9,79). A duração do tratamento variou de um a oito meses (média 3,6; DP 1,82), o número de sessões de três a 21 (média 11,2; DP 4,64). O tempo na docência variou de 36 a 456 meses (média 209,6; DP 102,67). Quanto ao tipo de disfonia identificada no início do tratamento, 20 $(51,2 \%)$ pacientes apresentavam disfonia funcional e 19 (48,7\%) organofuncional. Não houve casos de disfonias orgânicas na amostra avaliada.

As entrevistas foram realizadas ao telefone, por um fonoaudiólogo que não conheceu os pacientes no período do tratamento, baseadas em roteiro prévio constando de perguntas abertas, as quais foram elaboradas segundo a experiência clínica dos pesquisadores. As perguntas buscaram analisar a adesão a orientações fonoaudiológicas quanto ao comportamento vocal e realização de exercícios vocais após a alta do serviço. A entrevista semiestruturada constou das seguintes perguntas relacionadas à Categoria "Adesão às orientações":

- Quando você teve alta da Fonoaudiologia, o terapeuta recomendou que você tivesse alguns cuidados com a voz?

- Quais foram?

- Você os seguiu?

Os cuidados éticos foram tomados. Os pacientes foram esclarecidos quanto aos objetivos da pesquisa e aceitaram voluntariamente participar do estudo, expressando consentimento para uso das informações coletadas. Todos assinaram o Termo de Consentimento Livre e Esclarecido. A pesquisa foi aprovada pelo Comitê de Ética em Pesquisa da Universidade Federal de Minas Gerais (UFMG) sob o parecer ETIC 482/08.

Os resultados foram obtidos e analisados de acordo com a perspectiva da pesquisa qualitativa que incluiu a entrevista semi-estruturada e análise do conteúdo ${ }^{(10,14)}$ e posteriormente foram levantados a frequência do aparecimento de tais respostas, por meio de uma análise quantitativa.
As respostas obtidas ao telefone foram registradas no computador e, posteriormente, foram categorizadas e analisadas junto aos dados que estavam registrados nos prontuários dos pacientes, a fim de descrever o perfil dos sujeitos. As respostas dos pacientes quanto à adesão às orientações fonoaudiológicas foram classificadas nas seguintes categorias: presente (sendo cada tipo de orientação uma subcategoria) e ausente (Figura 1).

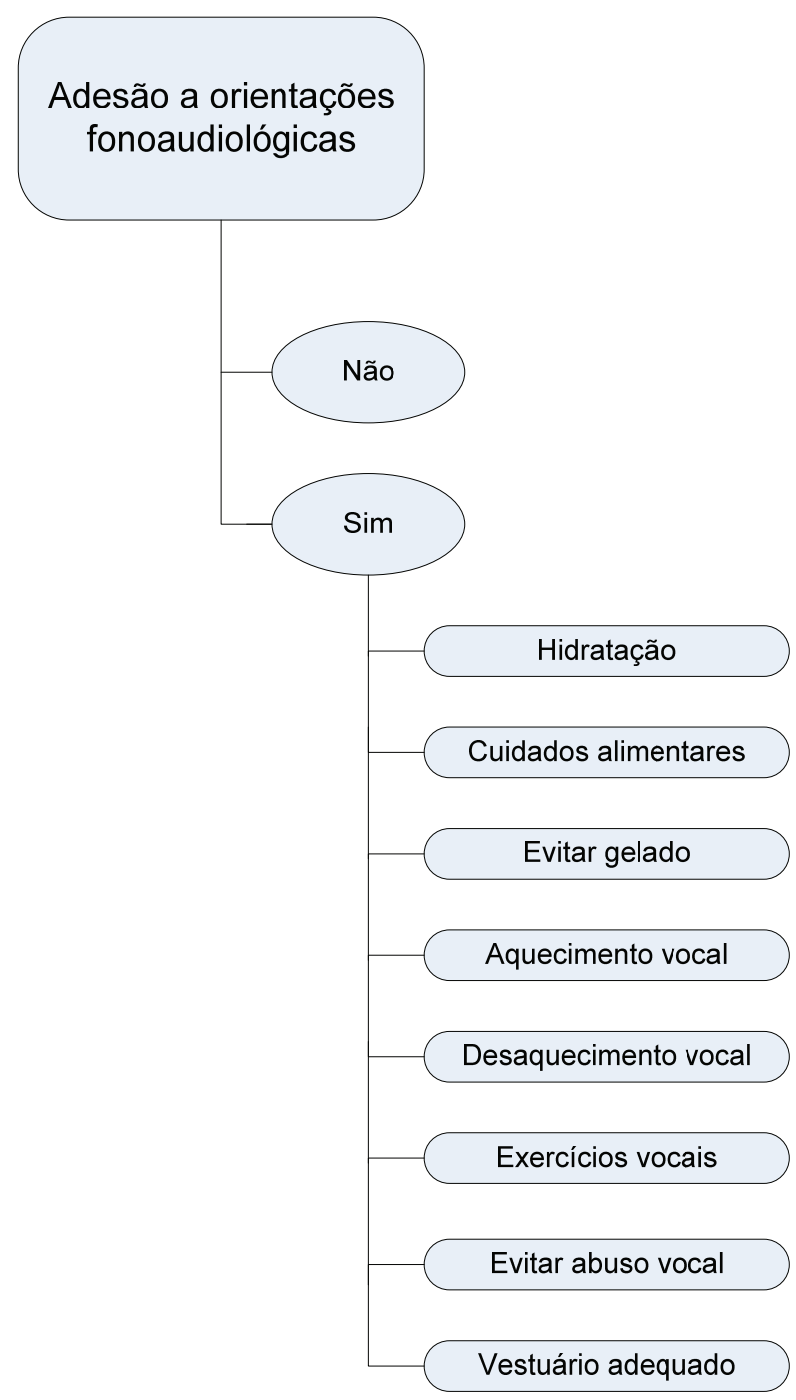

Figura 1 - Categoria e subcategorias de análise das respostas sobre a adesão às orientações fonoaudiológicas após alta do tratamento

\section{RESULTADOS}

Com relação à adesão às orientações fonoaudiológicas, a maioria dos pacientes referiu seguir sistematicamente as orientações fonoaudiológicas após a alta do tratamento de voz. As adesões às 
orientações mais prevalentes foram: hidratação, cuidados alimentares, evitar abusos vocais, realizar aquecimento vocal e exercícios vocais (Tabela 1).

O período entre a alta fonoterápica e a realização das entrevistas variou de um a 18 meses (média 7,6; DP 5,36), sendo que o tempo igual ou superior a seis meses foi correspondente a 23 casos $(58,9 \%)$ e, 16 pacientes $(41,02 \%)$ receberam alta fonoaudiológica no período de tratamento inferior a seis meses.

A maior parte dos entrevistados apresentou mais de uma resposta nesse item e algumas respostas foram transcritas (Figura 2).

Tabela 1 - Adesão às orientações fonoaudiológicas após a alta do tratamento

\begin{tabular}{lcc}
\hline & Número (N) & Porcentagem (\%) \\
\hline Sim & 34 & $87,1 \%$ \\
Parcialmente & 4 & $10,2 \%$ \\
Não & 1 & $2,5 \%$ \\
Hidratação & 31 & $81,5 \%$ \\
Cuidados alimentares & 24 & $63,1 \%$ \\
Evitar abusos vocais & 24 & $63,1 \%$ \\
Aquecimento & 18 & $47,3 \%$ \\
Exercícios vocais & 16 & $42,1 \%$ \\
Desaquecimento & 10 & $26,3 \%$ \\
Evitar gelado & 6 & $15,7 \%$ \\
Uso de vestuários adequados & 1 & $2,6 \%$ \\
\hline
\end{tabular}

\begin{tabular}{|c|c|}
\hline Tipo de cuidado com a voz & Exemplos de resposta \\
\hline Hidratação & $\begin{array}{l}\text { "... tá sempre hidratando as pregas vocais..." } \\
\text { "... foi orientado levar água para a sala de aula..." }\end{array}$ \\
\hline Cuidados alimentares & $\begin{array}{l}\text { "... comer maçã que é bom para a voz...” } \\
\text { "... procurar não ingerir alguns alimentos, como café e chocolate e } \\
\text { comer maçã." }\end{array}$ \\
\hline Evitar gelado & $\begin{array}{l}\text { "Muita água, nunca gelada e agora só tomo água natural". } \\
\text { "... não beber gelado, beber água mais a temperatura ambiente..." }\end{array}$ \\
\hline Aquecimento vocal & $\begin{array}{l}\text { "Seria, estar sempre fazendo os exercícios antes de começar a aula...." } \\
\text { "... faço exercícios de aquecimento..." }\end{array}$ \\
\hline Desaquecimento vocal & $\begin{array}{l}\text { "... fazer os exercícios de desaquecimento". } \\
\text { "... exercícios antes de começar e depois das aulas..." }\end{array}$ \\
\hline Exercícios vocais & $\begin{array}{l}\text { "Quando sentir alteração na voz é para fazer exercício, mas tem que } \\
\text { fazer exercício todo dia, né?" } \\
\text { "... tô fazendo aqueles exercícios do r e do s..." }\end{array}$ \\
\hline Vestuário adequado & “... não usar roupas muito justas e saltos, coisas que eu fazia muito”. \\
\hline Evitar abusos vocais & $\begin{array}{l}\text { "Evito falar em lugares muito barulhentos". } \\
\text { "... colocar os alunos mais próximos de mim para não falar muito alto..." } \\
\text { "Agora uso apito para não precisar de gritar". }\end{array}$ \\
\hline
\end{tabular}

Figura 2 - Exemplos de respostas dos pacientes classificadas quanto ao tipo de orientações fonoaudiológicas

\section{DISCUSSÃO}

Quando o foco do estudo é avaliação de adesão ao tratamento, os métodos de análise podem ser classificados em diretos e indiretos, sendo que não há um método considerado gold standard ${ }^{15}$. Os métodos diretos são baseados em técnicas analíticas que verificam, por meio de exames objetivos, 
a presença de marcadores químicos no organismo. Os métodos indiretos incluem entrevistas com os pacientes ${ }^{16}$. Levando-se em consideração o objeto de estudo, optou-se pela utilização do método indireto por meio de entrevistas. Vale ressaltar que este tipo de análise está sujeita a percepções individuais, portanto, estudos futuros com outros desenhos metodológicos são importantes para convalidar os resultados desta pesquisa.

A baixa adesão ao tratamento é um problema mundial, segundo a Organização Mundial de Saúde (OMS). Nos países desenvolvidos a adesão aos tratamentos de longa duração, na população geral, é em torno de $50 \%$, sendo que os países em desenvolvimento apresentam taxa de adesão muito abaixo da citada ${ }^{17}$.

No tocante à terapia vocal, a literatura refere uma taxa de $65 \%$ de abandono do tratamento ${ }^{18,19}$. Dos 76 sujeitos iniciais, $46 \%$ foram desligados do ambulatório, porcentagem menor do que a encontrada pelo estudo supracitado. Já em relação aos sujeitos que receberam alta, a grande maioria aderiu, de forma sistemática, às orientações fornecidas na fonoterapia. $O$ alto índice de adesão entre os pacientes que concluíram o tratamento discorda da literatura, que demonstra elevada taxa de ausência de adesão ao tratamento, mesmo no caso de doenças crônicas ${ }^{5-8}$.

Um estudo cujo objetivo era avaliar o efeito das orientações fonoaudiológicas, pesquisou 20 professoras que participaram de um curso sobre cuidados com a voz. Após 12 meses, foram enviados às participantes questionários referentes à qualidade de vida e aos benefícios conquistados no curso e foi constatado que os efeitos positivos se mantiveram. A manutenção das mudanças a longo prazo pode ser reflexo de uma maior conscientização sobre os problemas de voz e a produção correta da voz ${ }^{20}$.

Os resultados desta pesquisa são concordantes com a literatura ${ }^{20}$ e, a provável explicação do alto índice de adesão às orientações da terapia de voz após alta é que os pacientes que concluíram o tratamento já eram de certa forma, aderentes ao processo terapêutico, por terem solicitado atendimento fonoaudiológico e por concluírem o tratamento ${ }^{9}$. Além disso, provavelmente, a maior parte dos pacientes recebeu alta fonoaudiológica ao atingir o estágio de manutenção de acordo com o Modelo Transteórico e, portanto, já haviam adotado novos comportamentos, mantendo os cuidados relacionados à saúde vocal ${ }^{11}$. A adesão às mudanças comportamentais a longo prazo pode ser resultante de uma maior conscientização sobre a voz e sua correta produção 21,22 .

Quatro pacientes relataram seguir parcialmente as orientações e, provavelmente, se encontravam no estágio de preparação, no qual se comprometeram a modificar comportamentos vocais e foram experimentadas pequenas mudanças. Porém, um paciente não segue as orientações, provavelmente, em decorrência da estabilidade vocal alcançada na fonoterapia, não sente necessidade de manter cuidados vocais após a alta.

Nos casos de adesão às orientações, a maior parte dos professores relatou seguir orientações como hidratação, cuidados alimentares, controle de abusos vocais e realização de aquecimento vocal. Tais medidas são de extrema importância por prevenirem alterações laríngeas que conduzem a quadros disfônicos que culminam em afastamentos do trabalho. Sabe-se que mudar de comportamento nem sempre é fácil, requer conscientização e treinamento dirigido para a melhora da comunicação ${ }^{6,14}$. A maior prevalência da realização destes cuidados vocais pode estar relacionada ao fato destes comportamentos serem mais fáceis de serem seguidos e/ou pelo fato de os pacientes sentirem alívio imediato após sua realização. Pesquisas futuras poderão esclarecer por que os pacientes aderem a um tipo de orientação deixando de seguir outras.

O período entre a alta fonoterápica e a realização das entrevistas com os pacientes desta pesquisa variou de um a 18 meses, porém, não encontramos relação entre o tempo de alta e a adesão às orientações fonoaudiológicas. Estudos com um maior número de pacientes são necessários para se compreender melhor a existência ou não da interferência do tempo da alta fonoterápica na adesão a tais orientações.

Seria lúcido pensar que, pelo menos na experiência do AV-HC, a atuação fonoaudiológica tenha sido positiva para manter um adequado comportamento vocal, favorecendo a adesão as orientações fonoaudiológicas após a alta do tratamento.

\section{CONCLUSÃO}

Houve adesão às orientações fonoaudiológicas após a alta do tratamento vocal em docentes e as principais medidas realizadas foram hidratação, cuidados alimentares, controle de abusos vocais e realização de aquecimento vocal e de exercícios vocais.

\section{AGRADECIMENTOS}

A Pró-Reitoria de Pesquisa (PRPq) pelo fornecimento de bolsa de iniciação científica e ao Banco Santander pelo auxílio financeiro. 


\section{ABSTRACT}

Purpose: to investigate the adherence to voice therapy guidelines of teachers in municipal schools that were treated at the Voice Clinic of Hospital das Clínicas, Federal University of Minas Gerais, and that were discharged from voice therapy. Method: 39 patients treated at the Voice Clinic of Hospital das Clínicas, Federal University of Minas Gerais were interviewed by phone from August 2007 to December 2008. The interviews aimed to analyze the adherence to the guidelines after voice therapy discharge. Results: this study outcome indicates that 34 patients (87.1\%) report following the guidelines, four $(10.2 \%)$ do it partially and one $(2.5 \%)$ referred not following the guidelines. Conclusion: the majority of teachers adhere to the guidelines after voice therapy discharge. The outcome suggests that the voice therapy helps to maintain the standard of voice and a healthy vocal behavior.

KEYWORDS: Voice; Voice Disorders; Patient Compliance; Speech, Language and Hearing Science

\section{REFERÊNCIAS}

1. Bassi IB, Medeiros AM, Menezes LN, Teixeira LC, Assunção AA, Gama ACC. Health quality, self-percepted dysphony and dysphony diagnosed through clinical assessments in teachers. Journal of Voice, 2010 in press.

2. Kasama ST, Brasolotto AG. Percepção vocal e qualidade e vida. Pró-Fono 2007;19(1):19-27.

3. Ramig LO, Verdolini K. Treatment efficacy: voice disorders. J Speech Lang Hear Res. 1998;41:S101-16.

4. Niebudek-Bogusz E, Sznurowska-Przygocka B, Fiszer M, Kotyło P, Sinkiewicz A, Modrzewska M, Sliwinska-Kowalska M. The effectiveness of voice therapy for teachers. Folia Phoniatr Logop. 2008; 60 (3):134-41.

5. Silveira, LMC, Ribeiro VMB. Grupo de adesão ao tratamento: espaço de "ensinagem" para profissionais de saúde e pacientes. Interface Comunic., Saúde, Educ. 2004;9(16):91-104.

6. Rolim MO, Castro ME. Adesão às orientações fornecidas no Programa de Controle da Hipertensão: uma aproximação aos Resultados Padronizados de Enfermagem. Online braz. j. nurs. (Online); 2007;6(1).

7. Kramer JM, Hammill B, Anstrom KJ, Fetterolf D, Snyder R, Charde JP, Hoffman BS, LaPointe NA, Peterson E. National evaluation of adherence to B-blocker therapy for 1 year after acute myocardial infarction in patients with commercial health insurance. Am Heart J. 2006;152(3):454.

8. Barros ACM, Rocha MB, Helena ETS. Adesão ao tratamento e satisfação com o serviço entre pessoas com diabetes mellitus atendidas no PSF em Blumenau. Arq Catarin Med. 2008;37(1):54-62.
9. Portone C, Johns MM, Hapner ER. A review of patient adherence to the recommendation for voice therapy. J Voice. 2008;22(2):192-6.

10. Leer EV, Connor NP. Patient Perceptions of Voice Therapy Adherence. J Voice. 2010; 24 (4): 458-69.

11. Feer E,Hapner ER, Connor NP. Transtheoretical Modelo Health Behavior Change Appliedto Voice Therapy. J Voice. 2008;22(6):688-98.

12. J. Ruotsalainen, J. Sellman and L. Lehto et al., Systematic review of the treatment of functional dysphonia and prevention of voice disorders. Otolaryngol Head Neck Surg. 2008; 138: 557-65.

13. Behlau M, Madazio G, Feijó D, Pontes P. Avaliação de voz. In: Behlau M, organizador. Voz: o livro do especialista. Rio de Janeiro: Revinter; 2001. p. 69-73.

14. Graneheim UH, Lundman B. Qualitative content analysis in nursing research: concepts, procedures and measures to achieve trustworthiness. Nurse Educ Today. 2004;24:105-12.

15. Cramer, JA Mattson RH, Prevey ML, Séller RD \& Ouellette VL 1989. How often is medication taken as prescribed? JAMA. 1989; 261(22):3273-7

16. Brawley LR \& Culos-Reed N. Studying adherence to therapeutic regimens: overview, theories, recommendations.Controlled Clinical Trials. 2000; 21:156s-63s.

17. Adherence to long-therm therapies evidence for action. World Health Organization (WHO), 2003. Disponível em: http://www.who.int/chp/knowledge/ publications/adherence_report/en/

18. Portone-Maira C, Wise JC, Johns MM, Hapner EE. Differences in Temporal Variables between Voice Therapy Completers and Dropouts. J Voice. 2011; 25 (1): 62-6. 
19. Hapner E, Portone-Maira C, Johns MM. A study of voice therapy dropout. J Voice. 2009; 23 (3): 337-40.

20. Bovo R, Galceran M, Petruccelli J, Hatzopoulos S. Vocal problems among teachers: evaluation of a preventive voice program. $\mathrm{J}$ Voice. 2007;21(6):705-22.
21. Penteado RZ, Pereira IMTB. Qualidade de vida e saúde vocal de professores. Rev. Saúde Pública. 2007;41(2):236-43.

22. Jardim R, Barreto SM, Assunção AÁ. Condições de trabalho, qualidade de vida e disfonia entre docentes. Cad Saúde Públ. 2007;23(10):2439-61.

http://dx.doi.org/10.1590/S1516-18462011005000105

RECEBIDO EM: 21/02/2011

ACEITO EM: 15/04/2011

Endereço para correspondência:

Ana Cristina Côrtes Gama

Universidade Federal de Minas Gerais,

Faculdade de Medicina

Alfredo Balena,190/sala 3005 - Santa Efigênia

Belo Horizonte - MG

CEP: 30130-100

E-mail: anaccgama@gmail.com 